\title{
Simulating exposure-related behaviors using agent-based models embedded with needs-based artificial intelligence
}

\author{
Namdi Brandon ${ }^{1} \cdot$ Kathie L. Dionisio ${ }^{1} \cdot$ Kristin Isaacs $^{1} \cdot$ Rogelio Tornero-Velez $^{1} \cdot$ Dustin Kapraun $^{2} \cdot$ \\ R. Woodrow Setzer ${ }^{3} \cdot$ Paul S. Price ${ }^{1}$
}

Received: 13 November 2017 / Revised: 25 April 2018 / Accepted: 15 May 2018 / Published online: 21 September 2018

(c) The Author(s) 2018. This article is published with open access

\begin{abstract}
Exposure to a chemical is a critical consideration in the assessment of risk, as it adds real-world context to toxicological information. Descriptions of where and how individuals spend their time are important for characterizing exposures to chemicals in consumer products and in indoor environments. Herein we create an agent-based model (ABM) that simulates longitudinal patterns in human behavior. By basing the ABM upon an artificial intelligence (AI) system, we create agents that mimic human decisions on performing behaviors relevant for determining exposures to chemicals and other stressors. We implement the ABM in a computer program called the Agent-Based Model of Human Activity Patterns (ABMHAP) that predicts the longitudinal patterns for sleeping, eating, commuting, and working. We then show that ABMHAP is capable of simulating behavior over extended periods of time. We propose that this framework, and models based on it, can generate longitudinal human behavior data for use in exposure assessments.
\end{abstract}

Keywords: Agent-based model $\cdot$ Exposure-related behavior $\cdot$ Simulation $\cdot$ Artificial-intelligence

\section{Introduction}

Traditionally, exposure assessors and modelers obtain information about exposure-related behaviors by surveying individuals about their daily activities [1]. Surveyed individuals complete diaries that capture a range of activities over time [2-4]; the United States Environmental Protection Agency (U.S. EPA) has developed a database of such information for use in exposure assessment, called the Consolidated Human

Electronic supplementary material The online version of this article (https://doi.org/10.1038/s41370-018-0052-y) contains supplementary material, which is available to authorized users.

Paul S. Price

Price.PaulS@epa.gov

1 National Exposure Research Laboratory, Office of Research and Development, United States Environmental Protection Agency, RTP, NC, USA

2 National Center for Environmental Assessment, Office of Research and Development, United States Environmental Protection Agency, RTP, NC, USA

3 National Center for Computational Toxicology, Office of Research and Development, United States Environmental Protection Agency, RTP, NC, USA
Activity Database (CHAD) [5]. However, collecting representative amounts of survey data is difficult and labor-intensive, especially for durations longer than 1 day. In addition, surveys cannot collect information of which the individual is either unaware (e.g., use of products by another nearby individual or the behavior of children) or is too timeconsuming to report (e.g., amounts of each consumer product used in a given day). To avoid these difficulties, we have developed a method that models exposure-related behavior through use of agent-based models.

Agent-based models (ABMs) simulate the actions and interactions of autonomous agents within a system. Within the context of ABMs, the term "agent" represents a simulated being with agency and thus differs from the traditional definition of the term used in chemical risk assessments. The goal of an ABM is to gain explanatory insights into the behavior of complex processes by simulating the behavior of agents obeying a set of rules. Over time, the interaction between rules and agents can often lead to emergent behavior useful in modeling systems. ABMs combine elements of decision theory, computational sociology, and Monte Carlo methods, and are widely used in a range of research areas including sociology, biology, and ecology [6-12]. Here, we present a framework that uses methods from both the $\mathrm{ABM}$ and the artificial intelligence (AI) communities in 
Table 1 Components of the example ABM

\begin{tabular}{lllll}
\hline Need & Activity & Object & Environment & Mathematical model \\
\hline Rest & Sleep & Bed & Residence & Linear function \\
Hunger & Eat breakfast & Food & Residence & Linear function \\
Hunger & Eat lunch & Food & Residence and workplace & Linear function \\
Hunger & Eat dinner & Food & Residence & Linear function \\
Income & Work & Occupational objects & Workplace & Step function \\
Travel & Commute to work & Transport & Outdoors & Step function \\
Travel & Commute from work & Transport & Outdoors & Step function \\
\hline
\end{tabular}

order to model human behavior for simulating daily activities.

Needs-based AI is a method of decision-making used in ABMs based on agents' satisfying various competing needs [13]. In needs-based AI, the agent considers multiple motivations (e.g. the need to eat, sleep, commute, work, etc.) linked to corresponding actions. The decisions of an agent in choosing behaviors based on competing, time-varying needs generate the pattern of actions. By modeling how human needs change over time and how humans react to those needs, we are able to simulate human behavior. This approach allows the individual to have a large number of competing needs, and therefore can explain a wide range of behaviors.

The decision to use a needs-based AI framework within the proposed ABM is based on the concept that humans make their decisions to take actions in order to fulfill needs. That is, when a need is in an "unsatisfied" state, a person will attempt to perform an action that sends the respective need to a "satisfied" state. For example, if a person is hungry (a state where the need to eat is unsatisfied), a person will seek an action, eating, which in turn will bring the need to a satisfied state. The concept that needs govern human decision-making was first proposed by Maslow [14]. Maslow attempted to explain human behavior by organizing needs into a hierarchy from basic physiological needs that address survival to increasingly complex needs that address personal fulfillment. The proposed ABM addresses the basic physiological and societal needs that are a high priority for individuals. More specifically, we consider four behaviors in adults: sleeping, eating, working, and commuting. These behaviors are chosen because people spend large portions of their time engaged in them [15] and they are relevant for determining other exposure-related behaviors.

In what follows, we present the $\mathrm{ABM}$ components and the underlying algorithms. Afterwards, we describe a computational model of longitudinal variation in activity. We then apply the model to a case study that simulates week-long daily activity of a working adult. Lastly, we give a summary of our findings and provide a brief discussion concerning our research.

\section{Methods}

We now present the methods behind the ABM. We start by giving an overview of the model components. Next, we present how to model the decision-making process for choosing behaviors. Afterwards, we present the creation of a model of variation in longitudinal activity patterns followed by a description of parameterizing the longitudinal behavior.

\section{Model components}

The following is an overview of the various components used to implement a needs-based AI system. The reader is referred to Zubek [13] for a further explanation of the concepts presented.

\section{Agent}

An agent is an individual with control over its actions.

\section{Need}

A need is a time-varying characteristic of an agent and is the basis of the motivation of the agent to perform an action. Examples of needs include the desires to rest, to eat, to earn an income, and to change location. These needs are modeled in the example ABM and are referred to in the rest of this paper as Rest, Hunger, Income, and Travel, respectively. We assume that, without interventions (actions) on the part of the agent, the magnitudes of all needs change over time from a satisfactory state towards an unsatisfactory state. Reaching such a state triggers an agent to select an action that restores the respective need to a satisfactory state.

\section{Activity}

Activities are actions that an agent adopts in order to address unsatisfied needs. Activities have three properties. First, activities occur in specific environments (see Table 1). Second, activities begin at a specific point in time and have a 
finite duration. Third, an activity may impact multiple needs. In this version of the model, an agent can perform only one activity at a time. Unless otherwise stated, an activity inhibits decision-making during its duration.

\section{Linked activity}

Some activities are inherently coupled to other activities (e.g., commuting to or from work is coupled to the start or end of working, respectively). We denote these activities as linked activities.

\section{Object and environment}

Objects are items that an agent uses in order to perform an activity to address a need; the objects may be consumer products. Objects communicate with agents by advertising their value to individual agents. Environments are discrete collections of objects that can include one or more agents. An agent may move between environments, but can only be in one environment at any given moment. An agent may only interact with objects that share the agent's current environment.

The components of the model are summarized in Table 1.

\section{Satiation}

There are two quantities defining each need: its satiation $n$ expressed as a function of time $n(t)$ and its threshold parameter $\lambda$. The satiation is a measure of how satisfied an individual is with respect to a specific need. Satiation has a range $[0,1](n(t) \in[0,1])$ where a value of 0 indicates the most unsatisfied state and 1 the most satisfied state. Satiation decreases over time unless an agent performs an activity that causes the satiation to increase.

The threshold $\lambda$ controls how unsatisfied an agent must be in order for the agent to feel the desire to address the corresponding need. Alternatively said, the parameter $\lambda$ acts as a trigger to initiate an activity [16]. As long as the satiation exceeds $\lambda(n(t)>\lambda)$, an agent has no desire to address a need; the agent seeks activities only when $(n(t) \leq \lambda)$. This reflects the fact that people normally address needs only when they cross some threshold of satiation.

In this $\mathrm{ABM}$, satiation declines over time in two ways. The first type of decline depends on the amount of time since the need was last satisfied. Examples of such needs are Rest and Hunger. We can model people as fully energized when waking and becoming increasingly tired throughout the day. In this model the decay rate is assumed to be a constant. Mathematically, it's represented as

$$
m_{\text {decay }}=-\frac{1-\lambda}{\Delta t_{\text {decay }}},
$$

where $m_{\text {decay }}$ is the decay rate and $\Delta t_{\text {decay }}$ is the minimum amount of time for the need to go from a fully satisfied state to an unsatisfied state. In this case, the decaying behavior of the need is modeled as a linear function. However, the decaying behavior may be modeled as any monotonically decreasing function.

The second type of decline resembles step functions. Needs described by these satiation functions represent scheduled behaviors, where the agent has committed to perform an action (that will satisfy a long-term need) at a specific time (e.g., working for an organization or attending a school). Thus these functions depend on the current time of day, rather than the amount of time from a prior event. The satiation $n(t)$ for these needs may be mathematically modeled as

$n(t)=\left\{\begin{array}{l}1, t<t_{0} \\ \eta, t \geq t_{0}\end{array}\right.$

where $t$ is the current time and $t_{0}$ corresponds to the time of day that the need becomes unsatisfied. The parameter $\eta$ has value $0 \leq \eta \leq \lambda$ and is the value of satiation once the corresponding motivation is triggered.

The satiation may increase due to an action in two ways. For a linearly behaving need, the recovery rate of the satiation of a need associated with an action is a constant defined as

$m_{\text {recover }}=\frac{1-\lambda}{\Delta t_{\text {recover }}}$,

where $m_{\text {recover }}$ is the recovery rate and $\Delta t_{\text {recover }}$ is the minimum amount of time required to go from the unsatisfied state equal to $\lambda$ to a completely satisfied state $(n=1)$ as the result of performing an activity. We can mathematically express the required duration to achieve full recovery through such an action as

$$
\Delta t_{\text {activity }}=\frac{1-n(t)}{m_{\text {recover }}} .
$$

Second, if the need resembles a step function, an action may increase the satiation as

$$
n(t)=\left\{\begin{array}{l}
\eta, t<t_{0} \\
1, t \geq t_{0}
\end{array}\right.
$$

where $t_{0}$ is the time at which the need is satisfied.

Table 1 summarizes the mathematical model of each need modeled in the ABM. 
Fig. 1 Decay behavior of needs. (Left) The behavior of a need modeled by a linear function. (Right) The behavior of a need modeled by a step function

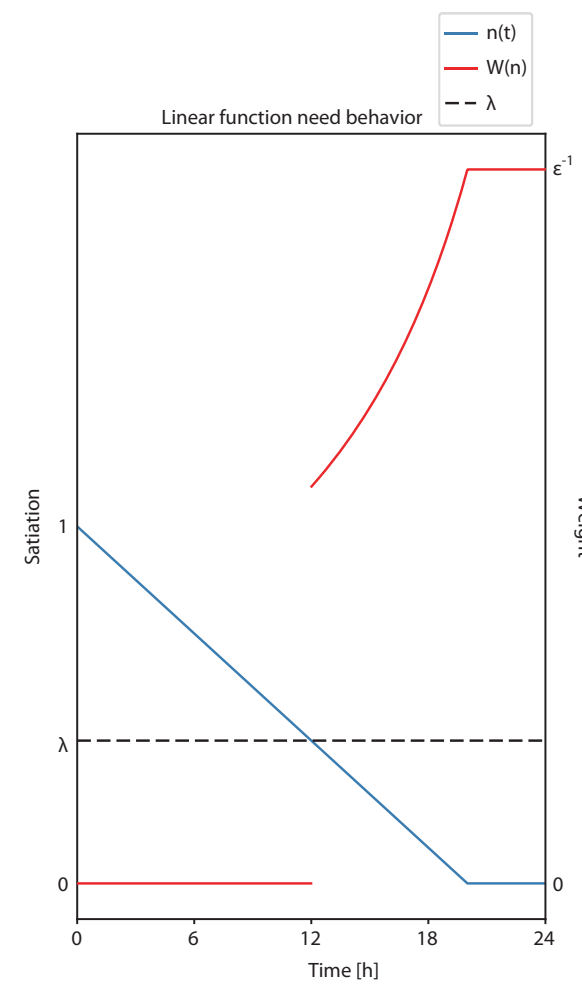

\section{Score}

Scores are a function of need-specific weights that measure the amount of urgency a need has. The weight function $W(n)$ has the property that it gives low values of satiation $n$ higher values of importance. That is, $W(n)$ is inversely proportional to $n$. This relationship captures the concept that lower values of $n(t)$ should correspond to more urgent desires. The weight function in this model is defined as

$W(n)=\left\{\begin{array}{c}0, n>\lambda \\ \frac{1}{n+\epsilon}, n \leq \lambda\end{array}\right.$

where $\in>0$ is a constant that prevents division by zero.

An agent chooses a particular activity by reviewing all activities advertised by the available objects and then by comparing the respective scores of all advertisements. First, note that performing an advertised activity should change an affected need $n(t)$ by some advertised amount $\delta$. When the advertised activity is completed in the future, the value of satiation will change. That is,

$n(t+\Delta t)=n(t)+\delta$.

Second, we base the advertised score not only on the agent's current state of need $n(t)$ but also on the agent's future state $\mathrm{n}(t+\Delta t)$, under the presumption that the respective activity of duration $\Delta t$ is chosen. Given the current state of a need, the advertised score $S(n)$ from an activity is

$$
S(n)=\left\{\begin{array}{c}
0, n>\lambda \\
W(n(t))-W(n(t+\Delta t)), n \leq \lambda .
\end{array}\right.
$$

First, note that if the satiation is above the threshold $\lambda$, no advertisement is given; hence, the score is 0 . Second, the use of the agent's future state in Eq. (7) allows for three types of outcomes for the score. The score will be positive if the activity increases the current value of the satiation; the score will be negative if the activity decreases the current value of the satiation; and the score will be zero if the activity does not change the current value of the satiation. In our framework, the agent chooses the activity with the highest positive score. If no activity fits that criteria, the agent "decides" to be idle (i.e., the agent does not perform any of the activities available in the ABM).

In general, once an activity is selected, the agent does not do anything else until the activity is completed. However, there are cases when it is useful for an agent to have the ability to interrupt a current activity in order to address a more pressing need. An example of this behavior is an agent stopping work in order to eat lunch. In the current model, the only activity that is interruptible is the work activity. The only activity that may interrupt working is eating lunch.

Figure 1 displays a representation of the interaction of satiation, weight function, and threshold for the two mathematical behaviors of needs. 


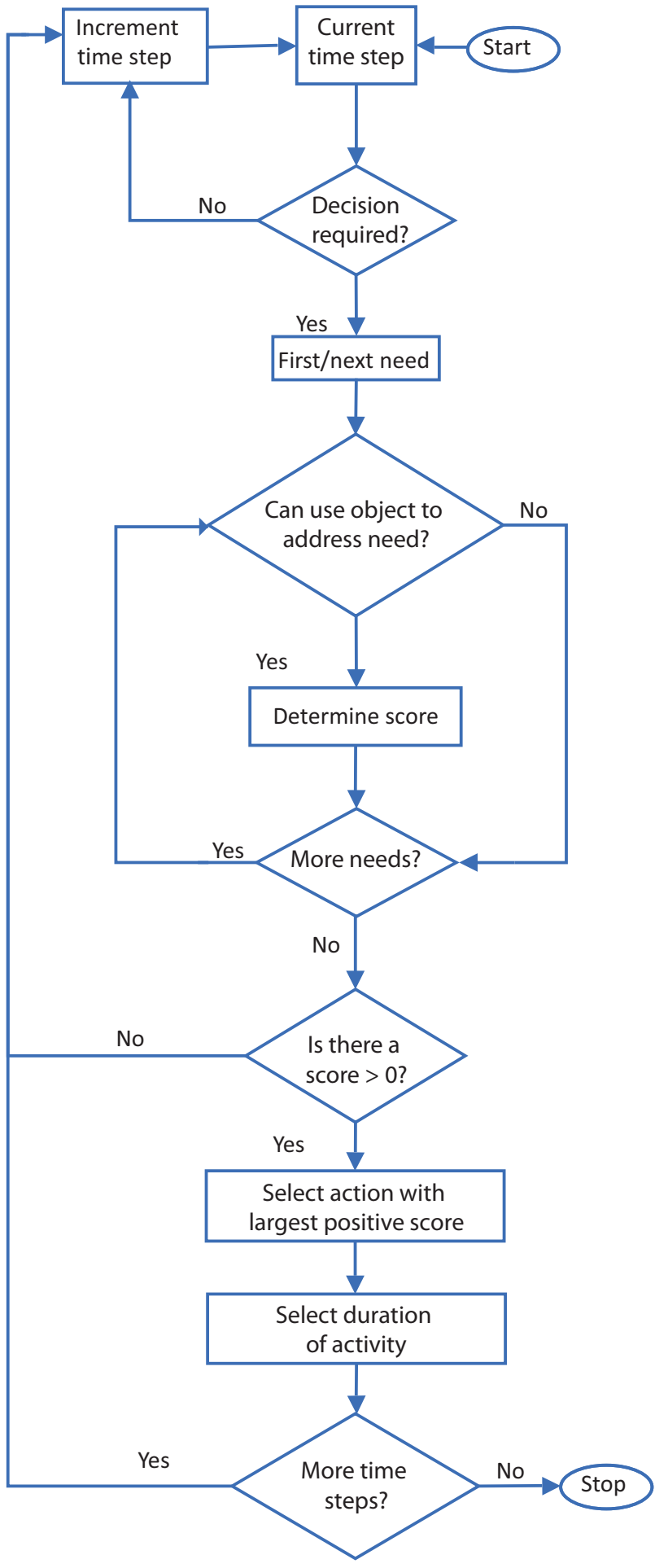

Fig. 2 AI for action decision-making

\section{Modeling the decision-making process for choosing behaviors}

The following describes how the previously discussed methodology fits within the application of the ABM. The ABM initializes by assigning the agent's fixed characteristics. This is necessary because many needs are dependent on the fixed characteristics of the agent. After initiation, the model moves an individual through time (1 minute at a time) by assigning the agent's actions based on the agent's needs over the simulation period.

Figure 2 illustrates the needs-based AI implemented in the ABM. At each time step, the AI allows the agent to go through the following decision-making process. First, the model begins by determining if the agent's current status permits decision-making. We assume that the activities sleeping, commuting, and eating cannot be interrupted. During such activities, the agent has no desire to consider other activities. If the agent is able to make a decision to start a new activity (i.e. the agent is currently idle or performing a behavior that can be interrupted), the model assesses each of the agent's needs. If a given need is unsatisfied, the model then determines if the respective need can be satisfied in the agent's current environment. In order to make this determination, the objects in the environment communicate their availability and usefulness with respect to the given need to the agent. In communicating with an agent, an object advertises all of its available abilities that address the given need. For the given activities, the score of performing the activity is determined by using Eq. (7). Once all of the objects advertise their scores for the respective need, the scores relevant for the next need are addressed. Finally, the model determines which need has the highest positive score, and the corresponding activity is started. If the agent has no needs below threshold that can be met (i.e., the agent is not yet unsatisfied) or has no score greater than 0 associated with it (i.e., there are no available activities within the environment that may increase the satiation of the agent), the agent remains idle until the next time step. The simulation continues until the total prescribed time for the simulation has elapsed.

\section{Creating a model of variation in longitudinal activity patterns}

We now describe the implementation of the above components and methods to create an algorithm to model individuals' behaviors over time. We call this algorithm the Agent-Based Model of Human Activity Patterns (ABMHAP). Currently, this model only includes four behaviors (sleeping, eating, commuting, and working). These four behaviors break down into seven distinct activities: sleeping, eating breakfast, commuting to work, working, eating lunch, commuting from work, and eating dinner. Every day the agent is expected to sleep and eat breakfast, lunch, and dinner. The agent is expected to commute and work 5 days a week (Monday-Friday). Eating lunch is modeled as being able to interrupt working. Commuting to and from work are linked to working. The morning commute ends with the 
beginning of work, and the evening commute starts with the end of work. All activities continue until the need is fully sated. The value of $\eta$ for commuting is set very low, resulting in a higher priority for leaving for work than for eating breakfast.

In the model, there are three environments: residence, workplace, and outdoors. The agent may move to or from the residence and outdoors and to or from the workplace and outdoors. The agent may not move from the residence directly to the workplace. In the residence, the objects required for sleeping and eating are present. In the workplace there are objects that allow working and eating. In the outside environment there is an object that allows movement to and from the workplace and residence.

\section{Modeling the decision-making process}

In ABMHAP, we denote the set of parameters start time, end time, and duration as the activity parameters for a given activity. Let $T_{\text {start }}, T_{\text {end }}$, and $\Delta T$ be random variables that describe the start time, end time, and duration, respectively, for a given activity. And let $t_{\text {start }}, t_{\text {end }}$, and $\Delta t$ be realizations of the random variables $T_{\text {start }}, T_{\text {end }}$, and $\Delta T$, respectively.

While each activity in the ABM has a start time, end time, and duration, the model defines two of the parameters for each non-linked activity. Activities that have a start time or an end time that are linked to another activity only require a duration. Table 2 specifies ABMHAP's parameterization for each activity where $\mathrm{X}$ indicates that the activity parameter is directly parameterized.

The sleep activity interacts with Rest's satiation $n(t)$ when the agent starts and ends the activity. Assume that at time $t_{0}$, the satiation for Rest $n\left(t_{0}\right) \leq \lambda$; and the agent decides to initiate the sleep activity. When the agent starts the activity, the algorithm uses Eq. (3) to calculate the recovery rate $m_{\text {recover }}$ and Eq. (4) to calculate $\Delta t_{\text {activity. }}$. Recall that if $n\left(t_{0}\right)<\lambda$, then the agent will sleep longer than the minimum recovery time (i.e., $\Delta t_{\text {activity }}>\Delta t_{\text {recover }}$ ). At time $\Delta t_{\text {end }}=\Delta t_{\text {start }}+\Delta t_{\text {activity, }}$, the agent ends the activity with Rest fully satisfied. The time for the next

Table 2 Display of parameters that define each activity

\begin{tabular}{llll}
\hline Activity & Start time & End time & Duration \\
\hline Sleep & $\mathrm{X}$ & $\mathrm{X}$ & \\
Eat breakfast & $\mathrm{X}$ & & $\mathrm{X}$ \\
Commute to work & & $\mathrm{X}$ & \\
Work & $\mathrm{X}$ & $\mathrm{X}$ & $\mathrm{X}$ \\
Eat lunch & $\mathrm{X}$ & & $\mathrm{X}$ \\
Commute from work & & $\mathrm{X}$ \\
Eat dinner & $\mathrm{X}$ & & \\
\hline
\end{tabular}

$\overline{\mathrm{X} \text { indicates which activity parameters are parameterized in the model }}$ sleep occurrence is determined by the time that the value of Rest's satiation $n\left(t_{0}\right)=\lambda$.

The "eat breakfast", "eat lunch", and "eat dinner" activities interact with Hunger's satiation $n(t)$ when the agent starts and ends the respective activity. What follows is an analysis for the eat breakfast activity; the eat lunch and eat dinner activities behave similarly.

Assume that at time $t_{0}$, Hunger's satiation $n\left(t_{0}\right) \leq \lambda$; and the agent decides to initiate the eat breakfast activity. Assume that the agent has the values $t_{\text {start }}$ and $\Delta t$ (see Table 2). When the agent starts the activity, the algorithm uses Eq. (3) to calculate the recovery rate $m_{\text {recover }}$ by using $\Delta t_{\text {recover }}=\Delta t$. The true duration of the activity is calculated from Eq. (4). At time $t_{1}=t_{0}+\Delta t_{\text {activity }}$, the agent ends the activity with Hunger fully satisfied. A new set of values is sampled for $t_{\text {start }}$ and $\Delta t$. Finally, the satiation decay rate $m_{\text {decay }}$ is calculated from Eq. (1) where $\Delta t_{\text {decay }}$ is the amount of time until the next meal starts (in this case of breakfast, the next meal is lunch).

The commute to or from work activity interacts with Travel's satiation $n(t)$ when the agent starts and ends the respective activity. What follows is an analysis for the commute to work activity; the commute from work activity behaves similarly.

Assume that at time $t_{0}$, Travel's satiation $n\left(t_{0}\right)=\eta \leq \lambda$; and the agent decides to initiate the commute activity. Assume that the agent has the value $\Delta t$ (see Table 2). When the agent starts the commute to work activity, the agent stays in the current activity until time $t=t_{0}+\Delta t$. At this time, the agent ends the activity with Travel being fully satisfied. Finally, a new value is determined for $\Delta t$ for the next commute to work activity occurrence.

The work activity interacts with Income's satiation $n(t)$ when the agent starts and ends the respective activity. Assume that at time $t_{0}$, Income's satiation $n\left(t_{0}\right)=\eta \leq \lambda$; and the agent decides to initiate the work activity. Assume that the agent has the values $t_{\text {start }}$ and $t_{\text {end }}$ (see Table 2). When the agent starts the work activity, the agent stays in the current activity until time $t=t_{\text {end. }}$. The agent ends the activity with Income fully satisfied. Finally, a new set of values is sampled for $t_{\text {start }}$ and $t_{\text {end }}$ for the next work activity occurrence.

Competing needs occur at several points in a day. First, when the agent wakes it has three possible activities: remain idle, eat breakfast, or commute to work. If breakfast is selected, upon its termination the agent may decide to be idle or commute to work. Commuting and work are linked activities. Thus the agent must start work after the morning commute is over and commute home after the workday is complete. During work the agent may continue to work or break for lunch. Upon arrival at home the agent may be idle, eat dinner, or go to sleep. 
Table 3 Example activity diary for a working adult

\begin{tabular}{lllllll}
\hline Day & Start time & End time & Duration & Activity & Weekday & Environment \\
\hline 0 & $23: 00$ & $08: 00$ & $09: 00$ & Sleep & Sunday & Residence \\
1 & $08: 00$ & $08: 15$ & $00: 15$ & Eat breakfast & Monday & Residence \\
1 & $08: 15$ & $09: 00$ & $00: 45$ & Commute to work & Monday & Outdoors \\
1 & $09: 00$ & $12: 00$ & $03: 00$ & Work & Monday & Workplace \\
1 & $12: 00$ & $12: 30$ & $00: 30$ & Eat lunch & Monday & Workplace \\
1 & $12: 30$ & $17: 00$ & $04: 30$ & Work & Monday & Workplace \\
1 & $17: 00$ & $17: 30$ & $00: 30$ & Commute from work & Monday & Outdoors \\
1 & $17: 30$ & $20: 00$ & $02: 30$ & Idle & Monday & Residence \\
1 & $20: 00$ & $20: 45$ & $00: 45$ & Eat dinner & Monday & Residence \\
1 & $20: 45$ & $23: 30$ & $02: 45$ & Idle & Monday & Residence \\
1 & $23: 30$ & $08: 00$ & $08: 30$ & Sleep & Monday & Residence \\
2 & $08: 00$ & $08: 20$ & $00: 20$ & Eat breakfast & Tuesday & Residence \\
\hline
\end{tabular}

Time is represented as hours:minutes
Table 3 illustrates an example output of 1 day's worth of an agent's activity diary.

\section{Parameterizing the longitudinal behavior}

Each activity parameter $X$ in Table 2 is modeled as belonging to its own truncated normal distribution (with width equal to one standard deviation) with mean $\mu$, and standard deviation $\sigma$, respectively. The values of $\mu$ represents an agent's long-term average behavior, and $\sigma$ represents an agent's day-to-day variation for the given activity parameter. Whenever an agent performs an activity, the activity parameters corresponding to the respective activity are sampled from the distributions defined by their values of $\mu$ and $\sigma$. The sampled values for the activity parameters in Table 2 are then used to determine the parameters in Eqs. (1)-(5) in order to determine the satiation levels for the individual's needs. This approach to parameterization is used to facilitate the use of existing survey data on human behaviors for calibrating ABMHAP.

\section{Code availability}

ABMHAP is coded in Python 3.5 [17]. More information about ABMHAP is found in the Supplementary Information.

\section{Results}

We now present a case study of the above methodology as it is implemented in ABMHAP. The exact values for $\mu$ and $\sigma$ for each activity parameter used in the example simulation are presented in Table 4. These values are chosen for demonstrative purposes.
Table 4 Numerical values used in ABMHAP example analysis

\begin{tabular}{|c|c|c|c|}
\hline Activity & $\begin{array}{l}\text { Start time } \\
\text { [hours] }\end{array}$ & $\begin{array}{l}\text { End time } \\
\text { [hours] }\end{array}$ & $\begin{array}{l}\text { Duration } \\
\text { [hours] }\end{array}$ \\
\hline Sleep & $\begin{aligned} \mu & =22 \\
\sigma & =0.5\end{aligned}$ & $\begin{array}{l}\mu=8 \\
\sigma=2.5\end{array}$ & \\
\hline Eat breakfast & $\begin{aligned} \mu & =8.25 \\
\sigma & =0\end{aligned}$ & & $\begin{aligned} \mu & =0.25 \\
\sigma & =0\end{aligned}$ \\
\hline Commute to work & & & $\begin{aligned} \mu & =0.5 \\
\sigma & =0\end{aligned}$ \\
\hline Work & $\begin{aligned} \mu & =9 \\
\sigma & =0\end{aligned}$ & $\begin{aligned} \mu & =17 \\
\sigma & =0.08\end{aligned}$ & \\
\hline Eat lunch & $\begin{array}{l}\mu=12 \\
\sigma=0.25\end{array}$ & & $\begin{aligned} \mu & =0.5 \\
\sigma & =0.17\end{aligned}$ \\
\hline $\begin{array}{l}\text { Commute from } \\
\text { work }\end{array}$ & & & $\begin{array}{l}\mu=1 \\
\sigma=0\end{array}$ \\
\hline Eat dinner & $\begin{aligned} \mu & =19 \\
\sigma & =0.17\end{aligned}$ & & $\begin{aligned} \mu & =0.75 \\
\sigma & =0.08\end{aligned}$ \\
\hline
\end{tabular}

ABMHAP was used to model one individual for a period of 7 days and $8 \mathrm{~h}$. The simulation started on Day 0, Sunday, at 16:00 with the agent in the residence environment and ended on Day 8, Monday, at 0:00. (The extra $8 \mathrm{~h}$ on day zero are required to properly set up the modeling of the initial sleep event on Day 0.) The results of the simulation, which can be called an "activity diary," are visualized in Fig. 3.

The simulation shows that ABMHAP is capable of producing longitudinal behavior patterns of a working adult that include day-to-day variation. Here, we have an agent who attempts to sleep around 22:00, wake around 8:00, eat breakfast around 8:15 for about $15 \mathrm{~min}$, eat lunch around 12:00 for about $30 \mathrm{~min}$, eat dinner around 19:00 for about 45 min, work approximately from 9:00-17:00, commute to work for $30 \mathrm{~min}$, and commute from work for $60 \mathrm{~min}$. In addition, ABMHAP captures the difference in behavior from workdays (Monday-Friday) and non-workdays 


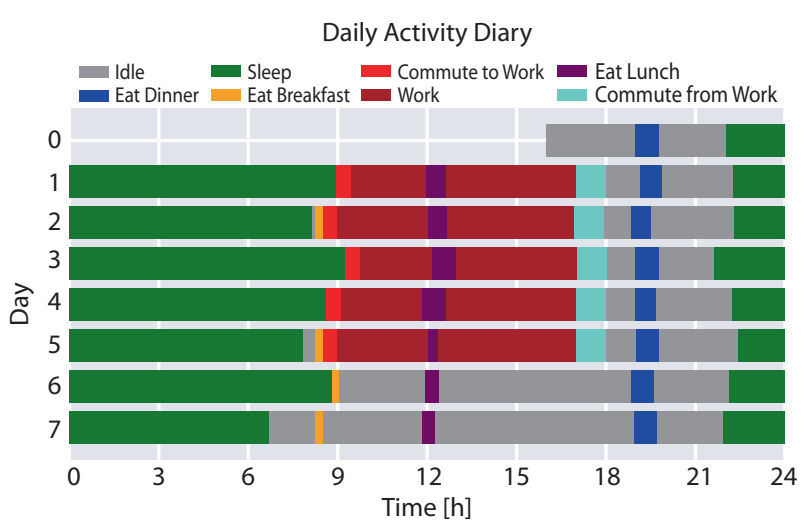

Fig. 3 Visualization of ABMHAP simulation output

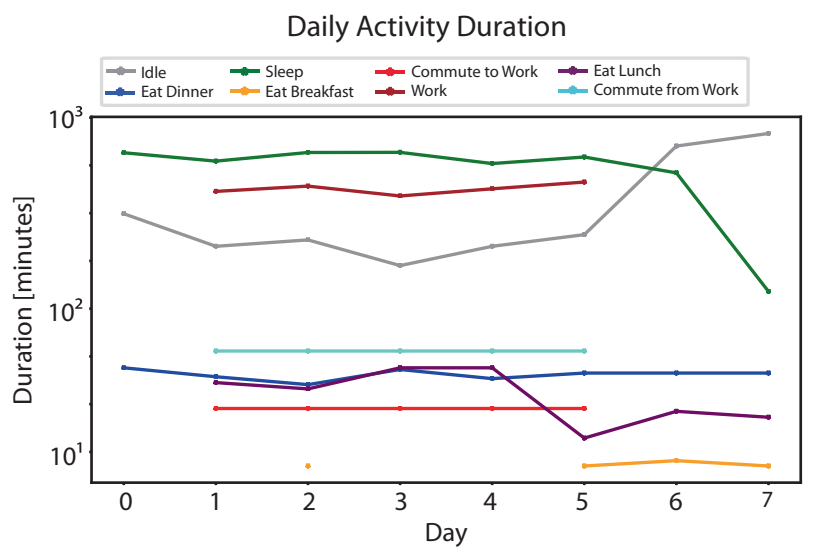

Fig. 4 Visualization of activity durations of an ABMHAP simulation. The durations are expressed in a $\log _{10}$ scale

(Saturday and Sunday), notably the absence of working and commuting on the weekends. The model is able to simulate day-to-day variation in the activities by having $\sigma \neq 0$ for the respective activity parameters. Figure 4 portrays the daily variation of activity duration. Without this variation, the schedule for each weekday or weekend would be exactly the same, which is unrealistic. People do not repeat their actions exactly from 1 day to the next.

In this example, we deliberately assign a large value of $\sigma$ in sleep end time to show how ABMHAP may handle competing needs in the morning. Figure 3 shows that on Days 1, 3, and 4, the large value of $\sigma$ in sleep end time causes the agent to wake up so sufficiently late that it causes the agent to skip breakfast. In these cases when waking up, the agent has competing unsatisfied needs Hunger and Travel (and in the case of Day 3, Income) to consider. Currently, the agent is parametrized to favor commuting to work rather than eating breakfast, which mimics how people may rush to work in order to minimize the amount of time that they may be late. The agent achieves this behavior by having Travel's satiation be set to a much lower value than Hunger's. This can be seen in Fig. 5, which shows how
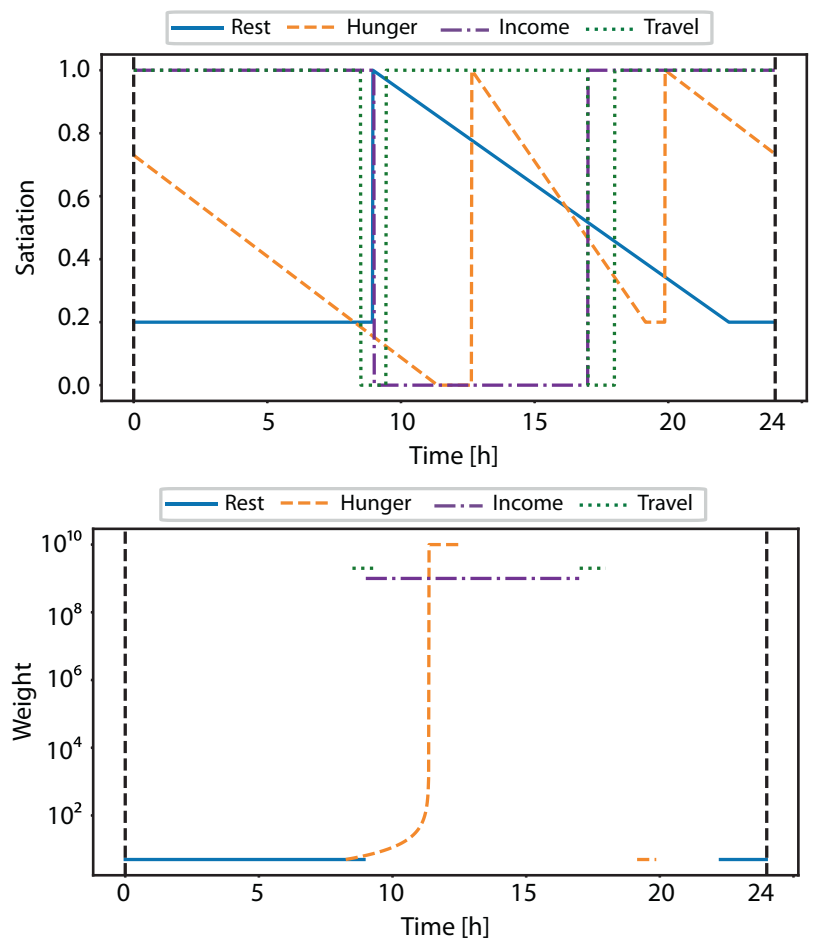

Fig. 5 The mathematical components involved for decision-making from the ABMHAP simulation on Day 1, Monday. (Top) The satiation values $n(t)$ for each need (recall the value of the threshold $\lambda=0.2$ ). (Bottom) The non-zero values of the weight function $W(n)$. The values are expressed in a $\log _{10}$ scale

both the satiation values and the weight function values change over time in Day 1 for each need. On Day 2 and Day 5 , the agent wakes up early enough that there is idle time between working and eating breakfast, causing the agent to eat breakfast and commute to work on time. On Day 6, the agent wakes up far after the expected time to eat breakfast. However, since the only unsatisfied need is Hunger at this point in time, the agent simply starts eating breakfast. Lastly on Day 7, the agent wakes up earlier than expected, and each need is addressed as soon as it becomes unsatisfied.

\section{Discussion}

The ability to simulate longitudinal patterns in exposurerelated behavior meet a critical need in the field of exposure assessment. It is difficult to perform longitudinal studies of exposure behaviors, and only a limited number of such studies are available. Unfortunately, the determination of the safety of chemicals requires information on the longitudinal patterns of exposure [18] which in turn require data on longitudinal patterns of human behavior [19]. The presented framework (and its implementation in ABHMAP) is proposed as a method for generating data that avoids the problems of measuring longitudinal patterns. This framework allows 
scientists to use behavior data on the frequency and duration of various types of activities that are collected in isolation and generate longitudinal behavior patterns (longitudinal activity diaries) as a coupled system of activities.

The current version of ABMHAP only addresses four types of behavior and does not address the more complex needs that are based on human interactions (child rearing, family activities, unscheduled group activities) nor does it address instances when demands of jobs or education supersede meals or sleep (working or studying late). However, the framework can be readily extended to consider these behaviors. The concepts defined in the framework (i.e., agents, objects that satisfy needs, environments, linked activities) and the proposed AI together present an approach that can be extended to any number of behaviors. The equations used to describe needs, weights, and scores are sufficiently flexible to allow the modeling of an individual's prioritization of competing needs over time. The parameter values in the equations can be informed by first principle arguments or calibrated with data from empirical surveys.

Exposures resulting from consumer product use can also be addressed by the framework. Consumer products are used as a result of individuals having needs that the products satisfy. Thus, the needs-based approach can be applied to the use of these products. Models that combine the four needs in ABMHAP and the consumer product-specific needs may be especially helpful in identifying times and durations when exposure events may occur. For example, many behaviors related to the use of consumer products that result in exposure events (e.g. cleaning kitchen floors with floor cleaner) would occur when an agent is present in the residence but not doing any of the four behaviors: sleeping, eating, working, and commuting. Use of other products, however, may happen concurrently with one of the four behaviors (e.g. putting on hand lotion while at work). In addition, ABMHAP's ability to generate activity patterns provides a unique opportunity for modeling interactions with many chemical sources (pollutants in ambient and indoor air) since it defines when an individual is in the workplace, outdoors, or at home.

\section{Summary and future Work}

The results suggest that needs-based AI underlying ABMHAP may be a suitable way of simulating the actions of working adults living in single-occupancy residences for the investigated behaviors. The model can incorporate data on day-to-day variation in behaviors and capture interactions between an individual's behaviors. As a next step in our research, we plan on calibrating ABMHAP with longitudinal survey data from $\mathrm{CHAD}$ and making longitudinal predictions of the four behaviors in adults and children. We do acknowledge that the case study only addresses a limited number of actions. In the future, this model could be extended to include behaviors involving the use of consumer products in order to help exposure-assessors identify times when these exposure-events may occur.

\section{Disclaimer}

The views expressed in this article are those of the authors and do not necessarily represent the views or policies of the U.S. Environmental Protection Agency.

Acknowledgements All funding was provided by the United States Environmental Protection Agency.

\section{Compliance with ethical standards}

Conflict of interest The authors declare that they have no conflict of interest.

Supplementary Information Documentation and a user guide for ABMHAP as well as the code itself may be found in GitHub (https:// github.com/HumanExposure/AgentBasedModel).

Open Access This article is licensed under a Creative Commons Attribution 4.0 International License, which permits use, sharing, adaptation, distribution and reproduction in any medium or format, as long as you give appropriate credit to the original author(s) and the source, provide a link to the Creative Commons license, and indicate if changes were made. The images or other third party material in this article are included in the article's Creative Commons license, unless indicated otherwise in a credit line to the material. If material is not included in the article's Creative Commons license and your intended use is not permitted by statutory regulation or exceeds the permitted use, you will need to obtain permission directly from the copyright holder. To view a copy of this license, visit http://creativecommons. org/licenses/by/4.0/.

\section{References}

1. Klepeis NE. An introduction to the indirect exposure assessment approach: modeling human exposure using microenvironmental measurements and the recent national human activity pattern survey. Environ Health Perspect. 1999;107:365-74.

2. Klepeis NE, Nelson WC, Ott WR, Robinson JP, Tsang AM, Switzer P, et al. The National Human Activity Pattern Survey (NHAPS): a resource for assessing exposure to environmental pollutants. J Expo Anal Environ Epidemiol. 2001;11:231-52.

3. Isaacs K, McCurdy T, Glen G, Nysewander M, Errickson A, Forbes $\mathrm{S}$, et al. Statistical properties of longitudinal time-activity data for use in human exposure modeling. J Expo Sci Environ Epidemiol. 2013;23:328-36.

4. Hertz-Picciotto I, Cassady D, Lee K, Bennett DH, Ritz B, Vogt R. Study of Use of Products and Exposure-Related Behaviors (SUPERB): study design, methods, and demographic characteristics of cohorts. Environ Health. 2010;9:54.

5. McCurdy T, Glen G, Smith L, Lakkadi Y. The national exposure research laboratory's consolidated human activity database. Int $\mathrm{J}$ Expo Anal Environ Epidemiol. 2000;10:566-78.

6. Heckbert S, Baynes T, Reeson A. Agent-based modeling in ecological economics. Ann N Y Acad Sci. 2010;1185:39-53. 
7. Hinkelmann F, Murrugarra D, Jarrah AS, Laubenbacher R. A mathematical framework for agent based models of complex biological networks. Bull Math Biol. 2011;73:1583-602.

8. Becher MA, Grimm VTP, Horn J, Kennedy PJ, Osborne JL. BEEHAVE: a systems model of honeybee colony dynamics and foraging to expolore multifactorial causes of colony failure. J Appl Ecol. 2014;51:470-82.

9. Chiacchio F, Mennisi M, Russo G, Motta S, Pappalardo F. Agentbased modeling of the immune system: NetLogo, a promising framework. Biomed Res Int. 2014;2014:907171.

10. Helbing D, Yu W, Opp KD, Rauhut H. Conditions for the emergence of shared norms in populations with incompatible preferences. PLoS ONE. 2014;9:e104207.

11. Khan B, Dombrowski K, Saad M. A stochastic agent-based model of pathogen propagation in dynamic multi-relational social networks. Simulation . 2014;90:460-84.

12. An G, Kulkarni S. An agent-based modeling framework linking inflammation and cancer using evolutionary principles: description of a generative hierarchy for the hallmarks of cancer and developing a bridge between mechanism and epidemiological data. Math Biosci. 2015;0:16-24.
13. Zubek R. Needs-based AI. In: Lake J, editor. Game Programming Gems 8. Boston, MA: Course Technology; 2011. p. 302-11.

14. Maslow AH. A theory of human motivation. Psychol Rev. 1943;50:370-96.

15. Moya J, Phillips L, Schuda L, Wood P, Diaz A, Lee R. et al. U. S. EPA. Exposure factors handbook: 2011 edition (final). Washington, DC, U.S.: Environmental Protection Agency; 2011.

16. De Silva L, Ekanayake H. Behavior-based robotics and the reactive paradigm. A survey. International workshop on data mining and artificial intelligence, Khulna, Bangladesh; 2008.

17. Python Software Foundation. Python Software Foundation Web site [Online]; 2017 [cited 19 July 2017]. Available from: https://www.python.org.

18. Zartarian V, Xue J, Glen G, Smith L, Tornero-Velez R. Quantifying children's aggregate (dietary and residential) exposure and dose to permethrin: application and evaluation of EPA's probabilistic SHEDS-multimedia model. J Expo Sci Environ Epidemiol. 2012;22:267-73.

19. Egeghy PP, Quackenboss JJ, Catlin S, Ryan PB. Determinants of temporal variability in NHEXAS-Maryland. J Expo Anal Environ Epidemiol. 2005;15:388-97. 